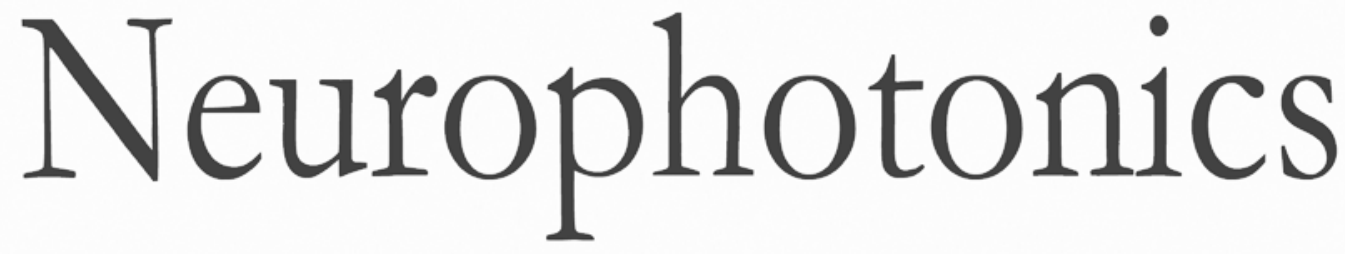

\title{
Mapping of real-time morphological changes in the neuronal cytoskeleton with label-free wide-field second- harmonic imaging: a case study of nocodazole
}

\author{
Marie E. P. Didier \\ Carlos Macias-Romero \\ Claire Teulon \\ Pascal Jourdain \\ Sylvie Roke
}

Marie E. P. Didier, Carlos Macias-Romero, Claire Teulon, Pascal Jourdain, Sylvie Roke, "Mapping of realtime morphological changes in the neuronal cytoskeleton with label-free wide-field second-

- - 2 harmonic imaging: a case study of nocodazole," Neurophoton. 6(4), 045006 (2019), 


\title{
Mapping of real-time morphological changes in the neuronal cytoskeleton with label-free wide-field second-harmonic imaging: a case study of nocodazole
}

\author{
Marie E. P. Didier, ${ }^{a}$ Carlos Macias-Romero, ${ }^{a}$ Claire Teulon, ${ }^{a}$ Pascal Jourdain, ${ }^{b}$ and Sylvie Roke ${ }^{a, *}$ \\ anstitute of Bioengineering, and Institute of Materials Science, School of Engineering, and Lausanne Centre for Ultrafast Science, École \\ Polytechnique Fédérale de Lausanne, Laboratory for fundamental BioPhotonics, CH 1015 Lausanne, Switzerland \\ ${ }^{b}$ Brain Mind Institute, École Polytechnique Fédérale de Lausanne, Laboratory of Neuroenergetics and Cellular Dynamics, CH 1015, \\ Lausanne, Switzerland
}

\begin{abstract}
We demonstrate the use of wide-field high-throughput second-harmonic (SH) microscopy for investigating cytoskeletal morphological changes on the single-cell level. The method allows for real-time, in vitro, label-free measurements of cytoskeletal changes that can, under certain conditions, be quantified in terms of orientational distribution or in terms of changes in the number of microtubules. As SH generation is intrinsically sensitive to noncentrosymmetrically structured microtubules, but not to isotropic or centrosymmetric materials, we use it to probe the microtubule structure in the cytoskeleton when it undergoes dynamic changes induced by the application of nocodazole, a well-known microtubule-destabilizing drug that reversibly depolymerizes microtubules. In addition, the orientational directionality of microtubules in neurites and cell bodies is determined label-free using SH polarimetry measurements. Finally, we use spatiotemporal SH imaging to show label-free, real-time nocodazole-induced morphological changes in neurons of different age and in a single axon. $\odot$ The Authors. Published by SPIE under a Creative Commons Attribution 4.0 Unported License. Distribution or reproduction of this work in whole or in part requires full attribution of the original publication, including its DOI. [DOI: 10.1117/1.NPh.6.4.045006]
\end{abstract}

Keywords: second-harmonic generation; multiphoton imaging; microtubule dynamics; spatiotemporal mapping; nocodazole; label-free.

Paper 19022R received Mar. 18, 2019; accepted for publication Sep. 23, 2019; published online Nov. 7, 2019.

\section{Introduction}

Microtubules are highly dynamic polymers ${ }^{1}$ that constitute a major component of the cytoskeletal network of neuronal cells. They are versatile structures capable of polymerizing and depolymerizing $^{2,3}$ and play an essential role in morphogenesis and are part of numerous cytoplasmic structures. ${ }^{4}$ Microtubules are spatially noncentrosymmetric hollow cylinders that are composed of $\alpha / \beta$-tubulin dimmers. ${ }^{5,6}$ In microtubules, the $\alpha / \beta$ tubulin dimer composition induces a dipole along the axial direction of the axonal microtubules' bundles. In axons of morphologically polarized neurons, microtubules are uniformly oriented in tightly packed bundles. ${ }^{7}$ In contrast, a less uniform directional distribution is present in dendrites. ${ }^{8,9}$ To understand the complexity, growth, maintenance, and remodeling of the microtubule bundles and thus the cytoskeleton, knowledge of the regulation of microtubule dynamics is important. Commercially available drugs exist to investigate and study microtubule dynamics. In this respect, nocodazole is a valuable tool. ${ }^{10}$ Nocodazole is a microtubule-specific drug ${ }^{11}$ that causes microtubule depolymerization by binding to free tubulin dimers and preventing them from incorporating into microtubules. ${ }^{12}$ Used at appropriately high concentration, nocodazole interrupts the microtubule polymerization behavior in a reversible way: ${ }^{13-15}$ after washout, microtubule polymerization occurs without affecting their initial orientation. Nocodazole is often used to probe instability and to uncover microtubule regulation mechanisms

*Address all correspondence to Sylvie Roke, E-mail: sylvie.roke@epfl.ch in combination with immunofluorescent methods. ${ }^{13}$ Tracking the dynamics of microtubules in vitro was first achieved in 1986 with dark-field differential interference contrast microscopy. ${ }^{16}$ In addition, techniques involving immunoelectron microscopy and fluorophore-tagged proteins have been used to visualize intrinsic and induced microtubule dynamics. ${ }^{17-21}$ More recently, optogenetic modifications or cloned proteins expression in mice $^{22}$ and differential interference contrast imaging methods ${ }^{23}$ have been used for the investigation of the cytoskeleton dynamics.

The necessity to tag microtubules with a label has disadvantages: the difficulty to attach the label specifically and permanently to microtubules, the photo instability induced by the use of a fluorophore, and the harsh interaction of the fluorophore on the chemical reaction of interest. ${ }^{24,25}$ In addition, genetic manipulation or differential microscopy has disadvantages from a clinical perspective or lack in component specificity.

An alternative for microtubule imaging has been recognized and demonstrated in the form of second-harmonic ( $\mathrm{SH}) \mathrm{mi}-$ croscopy. ${ }^{26-28}$ In a SH process, two near-infrared photons with frequency $\omega$ and field strength $E(\omega)_{j, k}$ (with polarization states $j$ or $k$ ) combine into one photon that has the double frequency $2 \omega$. The optical contrast of a SH microscope is determined by the spatial organization of molecules: a centrosymmetric or isotropic arrangement of molecules does not give rise to any coherent $\mathrm{SH}$ emission, while a polar arrangement of molecules does emit SH photons. ${ }^{29}$ This symmetry selection rule generates a unique specificity to noncentrosymmetric structures. Since microtubules are polar, they generate SH light, whereas 
cytoskeletal structures, such as actin filaments, do not. ${ }^{26}$ The SH emission also strongly depends on the way and the geometry in which microtubules are organized. The intensity in polarization state $k\left[I(2 \omega)_{i}\right]$ measured in SH experiment is given by the absolute square of the second-order nonlinear polarization $P_{i}^{(2)}(2 \omega)$ :

$$
\begin{aligned}
& I(2 \omega)_{i} \sim\left|P_{i}^{(2)}(2 \omega)\right|^{2} \quad \text { and } \\
& \quad P_{i}^{(2)}(2 \omega)=\epsilon_{0} \chi_{i j k}^{(2)}(2 \omega) E_{j}(\omega) E_{k}(\omega) .
\end{aligned}
$$

Here, $P_{i}^{(2)}(2 \omega)$ can be understood as a charge oscillation in the material that emits photons at the double frequency. The second-order susceptibility $\chi_{i j k}^{(2)}(2 \omega)$ is the material response and is given by the orientational average of the responses of the molecular building blocks that make up the material (microtubules); it is defined by the molecular hyperpolarizability tensor $\beta_{a b c}^{(2)}(2 \omega)$, where $a, b$, and $c$ are the molecular symmetry axes. For the following derivation, we assume that the microtubules are in plane and that due to the wide-field illumination configuration of our imaging technique, the paraxial approximation is valid. For these conditions, the second-order susceptibility is given as

$$
\begin{aligned}
\chi_{i j k}^{(2)}(2 \omega)= & \frac{N}{\epsilon_{0}} \sum \beta_{a b c}^{(2)}\left\langle R_{a i}(\psi, \theta, \phi) R_{b j}(\psi, \theta, \phi)\right. \\
& \left.\times R_{c k}(\psi, \theta, \phi) f(\psi, \theta, \phi)\right\rangle_{\psi, \theta, \phi},
\end{aligned}
$$

which is the orientational average of the hyperpolarizability tensor elements whereby $f(\psi, \theta, \phi)$ is the orientational distribution function, $\psi, \theta$, and $\phi$ are the Euler angles, ${ }^{30}$ and $N$ represents the number of microtubules. Based on the cylindrical symmetry of the system, one assumes spatial isotropy around two angles $(\psi, \phi)$ and directionally around only one orientational angle $(\theta)$. For a nonresonant optical process (involving only virtual energy states), there are only two nonzero ${ }^{31-34}$ $\chi_{i j k}^{(2)}(2 \omega)$ tensor elements, namely $\chi_{y y y}^{(2)}(2 \omega)$ and $\chi_{y x x}^{(2)}(2 \omega)$. Considering this analysis, one can measure the orientational angle of microtubules in a structure by measuring different polarization combinations. One way to obtain the tilt angle of a microtubule segment at a single pixel is to compute the ratio: $:^{31-34}$

$$
\frac{I(2 \omega)_{Y Y Y}}{I(2 \omega)_{X X X}}=\tan ^{2}(\theta) .
$$

The $X$ and $Y$ axis are perpendicular to the optical axis $Z$, and the $Z$ axis is parallel to the second harmonic $k$-vector. This analysis, thus, provides a way to determine the microtubule orientation on a pixel-by-pixel basis. It does not allow determining the directionality of microtubules. In Ref. 35, such directionality information is given using a combination of $\mathrm{SH}$ and two-photon fluorescence from labels. Interferometric $\mathrm{SH}$ imaging provides such label-free information, as shown in Ref. 36. This method has, however, not yet been applied to neuroimaging. Further, it can be seen from Eqs. (1)-(3) that the SH intensity depends quadratically on the number of microtubules if these are aligned along the same axis. This quadratic dependence is referred to as spatial coherence. This means that axons that have microtubules organized unidirectionally will emit a much stronger SH intensity than structures with a bidirectional orientation of microtubules, allowing for identification of the axon by means of the intensity. ${ }^{37}$ Thus, in principle, SH imaging allows for identification of different morphological parts of the cytoskeleton, and SH polarimetry can be used to determine orientational distributions of noncentrosymmetric structures. As SH imaging requires no immunostaining, such information can be obtained label-free in living neurons in real time. Unfortunately, the relative inefficiency of the nonlinear SH generation process compared to linear fluorescence has prohibited the widespread use of scanning confocal SH imaging ${ }^{38}$ to map neuronal microtubulebased cytoskeleton and dynamic processes in real time. ${ }^{26,39,40}$

Here, we explore the use of femtosecond, wide-field, medium repetition rate $\mathrm{SH}$ imaging ${ }^{41}$ for dynamic neuroimaging. The method combines an increase in throughput of 3 orders of magnitude of the SH imaging process ${ }^{41,42}$ with low photodamage. ${ }^{43}$ We use the well-known effects of nocodazole on microtubules as an example of a system where spatiotemporal changes are expected. We determine the orientational distribution of microtubules and map the relative intensity of different morphological structures and perform in-vitro imaging of the effects of nocodazole on the axon of a corpus of neurons at different stages of maturity. We observe an overall depletion and recovery of the $\mathrm{SH}$ intensity upon nocodazole application and washout, consistent with the expected reversible nocodazolemicrotubules interactions. We then demonstrate spatiotemporal dynamic SH imaging during and after nocodazole application using image acquisition times of $250 \mathrm{~ms}$. The images display a nonuniform degradation and structural recovery of the axonal microtubules. Assuming that the orientational distribution within the axon is unaffected by nocodazole, we convert the SH intensity changes into a change in the number of microtubules present in the structures and show the possibility of our imaging technique to image cytoskeletal structural changes label-free.

\section{Materials and Methods}

\subsection{Cell Culture}

Primary cultures of cortical neurons were prepared from E17 OF1 mice embryos of either sex. Briefly, embryos were decapitated and cortices were removed under a dissecting microscope and collected in a small Petri dish in phospate-buffered salineglucose (PBS-glucose). A single-cell suspension was obtained by gentle agitation with a fire-polished Pasteur pipette in Neurobasal Medium supplemented with B27 and GlutaMAX (Invitrogen, phenol red-free). Cells were plated at an average density of 15,000 cells $/ \mathrm{cm}^{2}$ in supplemented Neurobasal Medium on polyornithine-coated glass coverslips (20-mm diameter). After 3 to $4 \mathrm{~h}$, coverslips were transferred to dishes with supplemented Neurobasal Medium. Neurons were maintained at $37^{\circ} \mathrm{C}$ in a humidified atmosphere of $95 \%$ air $/ 5 \% \mathrm{CO}_{2}$ and were used between 2 and 22 days in vitro (DIV).

\subsection{Nocodazole Treatment}

Nocodazole was purchased from Sigma Aldrich (Buchs, Switzerland). A stock solution diluted in dimethyl sulfoxide (DMSO) was prepared with a concentration of $5 \mathrm{mg} / \mathrm{ml}$ and then diluted in tissue culture medium at a ratio of 1:500 for a final concentration of $10 \mu \mathrm{g} / \mathrm{ml}$. The tissue culture medium was prepared fresh prior to the experiment and contained the following concentrations (in millimolar) $140 \mathrm{NaCl}, 3 \mathrm{KCl}$, $3 \mathrm{CaCl}_{2} \cdot 2 \mathrm{H}_{2} \mathrm{O}, 2 \mathrm{MgCl}_{2} \cdot 6 \mathrm{H}_{2} \mathrm{O}, 5 \mathrm{mM}$ glucose, and $10 \mathrm{mM}$ 
(a)

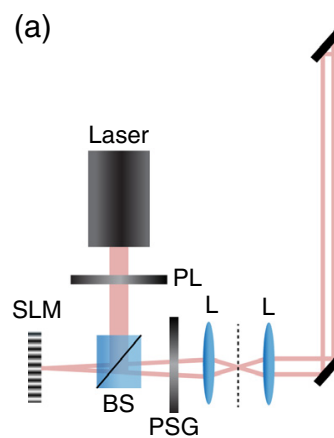

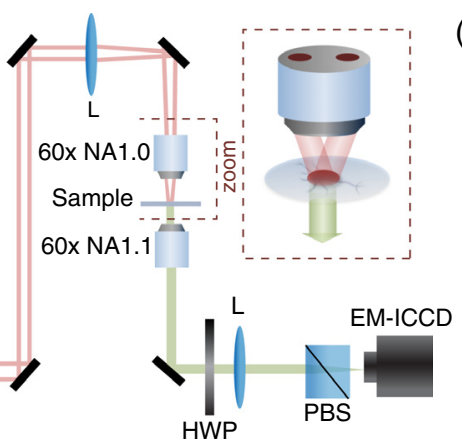

(b)

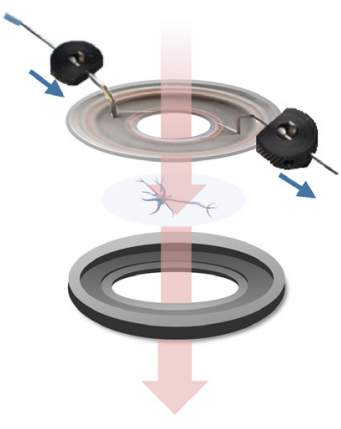

Fig. 1 Imaging configuration. (a) Schematic representation of the SH microscope, with optical components: SLM, polarizer (PL), beam splitter (BS), polarization state generator (PSG), achromatic lens (L), dichroic beam splitter (DB), NA, HWP, and polarizing BS (PBS). (b) Layout of the sample holder, with bottomed culture coverslips inserted in a low-profile, open bath chamber from Warner Instruments (Series 40 Quick Change Imaging Chambers RC-40LP).

Hepes (solution denoted as HEPES solution in this work). All chemicals were purchased from Sigma Aldrich. Nocodazole containing medium and the drug-free tissue culture medium were warmed to $37^{\circ} \mathrm{C}$ before using it at room temperature. Recordings of $\mathrm{SH}$ movies started a few seconds after the drug-containing medium was injected in the neurons-containing chamber and were also performed at room temperature. After 2 to $10 \mathrm{~min}$, the drug-containing solution was removed from the sample chamber by pipetting and replaced with a drug-free tissue culture medium. This tissue culture medium was replaced three times following the same procedure by pipetting and reinjection of medium to remove any nocodazole residue during the recovering period. The recording of the recovery experiment started after the third rinse and lasted for 10 to 20 min depending on the experiment.

\subsection{Second-Harmonic Imaging System}

The imaging system is sketched in Fig. 1(a) and is described in detail in Ref. 41. It is composed of a light source, a Yb: KGW amplified laser (Pharos Light Conversion) that delivers $200 \mathrm{kHz}, 1036 \mathrm{~nm}, 168 \mathrm{fs}$ laser pulses. The pulse energy delivered to the sample is $36 \mu \mathrm{J}$ and shaped into a elliptical illumination spot size with an FWHM of $150 \mu \mathrm{m}$ in the sample plane, reaching a fluence of $2.15 \mathrm{~mJ} / \mathrm{cm}^{2}$ and a peak intensity of $12.79 \mathrm{GW} / \mathrm{cm}^{2}$. To reach this type of focus, the laser beam illuminates a spatial light modulator (SLM, Phase Only Microdisplay 650 to $1100 \mathrm{~nm}$, with a fill factor of 93\%, $1920 \times 1080$ pixels, and a $8-\mu \mathrm{m}$ pixel pitch from HOLOEYE Photonics) under normal incidence. The SLM is used as a reflective diffraction grating and generates a grayscale hologram image of a rotatable slit pattern. The pattern is filtered in the Fourier plane of a lens ( $f=20 \mathrm{~cm}$, Thorlabs) placed after the SLM, such that only the 1 st and -1 st orders are retained. These two beams hit the back focal plane of a top objective [Olympus LUMPLFLN 60x1.0 numerical aperture (NA) water immersion objective] and are then imaged onto the sample plane such that the sample is illuminated with two wide-field plane waves. The opening angle between these beams is tunable by varying the grating spacing period on the SLM. The polarization of the two incoming beams is controlled by a zero-order half-waveplate (HWP) (Thorlabs, WPH05M-1030). The generated SH light was collected in the forward transmission direction using a high NA Olympus, LUMFLN 60x1.1 NA objective and analyzed with an analyzer placed in the collection path after a $515-\mathrm{nm}$ bandpass filter (Omega Optical, 10-nm bandwidth). The SH imaging was performed with a gated detection on a backilluminated electron-multiplying intensified charge-couple device (Bf GEN III, EM-ICCD, PiMax4, Princeton Instruments) with a $512 \times 512$ pixels chip. SH images were acquired with an acquisition time of $0.25 \mathrm{~s}$ each, with the electron multiplier gain set to 4000 and an accumulation parameter of 50,000 on the camera chip. The microscope also integrates a path for phase contrast (PC) imaging with white light.

\subsection{SH Imaging Experiments}

Coverslips with plated neurons were inserted in a Quick Change Imaging Chamber from Warner Instruments (Series 40 RC41LP). As sketched in Fig. 1(b), the bottom of the imaging chamber is formed by the glass coverslip with the plated neurons, on top of which a polycarbonate ring was gently placed. This imaging chamber contains a maximum of $3 \mathrm{~mL}$ of liquid and allows a rapid exchange of liquid, a short working distance, and an open bath. A constant flow of extracellular solution with $140 \mathrm{mM} \mathrm{NaCl}, 3 \mathrm{mM} \mathrm{KCl}, 3 \mathrm{mM} \mathrm{CaCl} 2 \cdot 2 \mathrm{H}_{2} \mathrm{O}$, $2 \mathrm{mM} \mathrm{MgCl} 2 \cdot 6 \mathrm{H}_{2} \mathrm{O}, 5 \mathrm{mM}$ glucose, and $10 \mathrm{mM}$ Hepes was used at a flow rate of $1 \mathrm{~mL} / \mathrm{mn}$ associated with a suction speed of $1.2 \mathrm{~mL} / \mathrm{mn}$ to provide the neurons with fresh solution and a laminar flow in the chamber. Prior to each imaging experiments, all neurons were checked for viability according to the protocol described in the supplementary information.

\section{Results and Discussion}

\subsection{Orientational Distribution of Microtubules}

Figure 2(a) shows a PC image of a cultured neuron at DIV13. The cell body and two neurites that are labeled 1 and 2 are visible in the image. Figure 2(b) shows the SH image recorded with two orthogonal polarization combinations (color coded with green for YYY and red for XXX polarized beams). The inset in Fig. 2(c) shows the application of Eq. (3) on the obtained data in Fig. 2(b). Neurite 1 is oriented mainly along the $X$ direction (90 deg), and neurite 2 is mainly oriented along the $Y$ direction, indicated by the arrows. Figure 2(c) shows the angular distribution for neurites 1 and 2 extracted from Eq. (3), using the arrows in Fig. 2(b) as reference $(0 \mathrm{deg})$. Neurite 1 displays a narrow angular distribution of microtubule segments, while neurite 2 has a broad orientational distribution that is not directed along the growth direction. Both distributions can be fitted with 


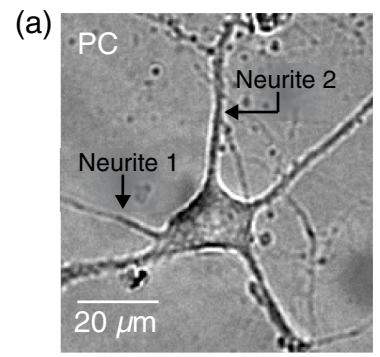

(b)
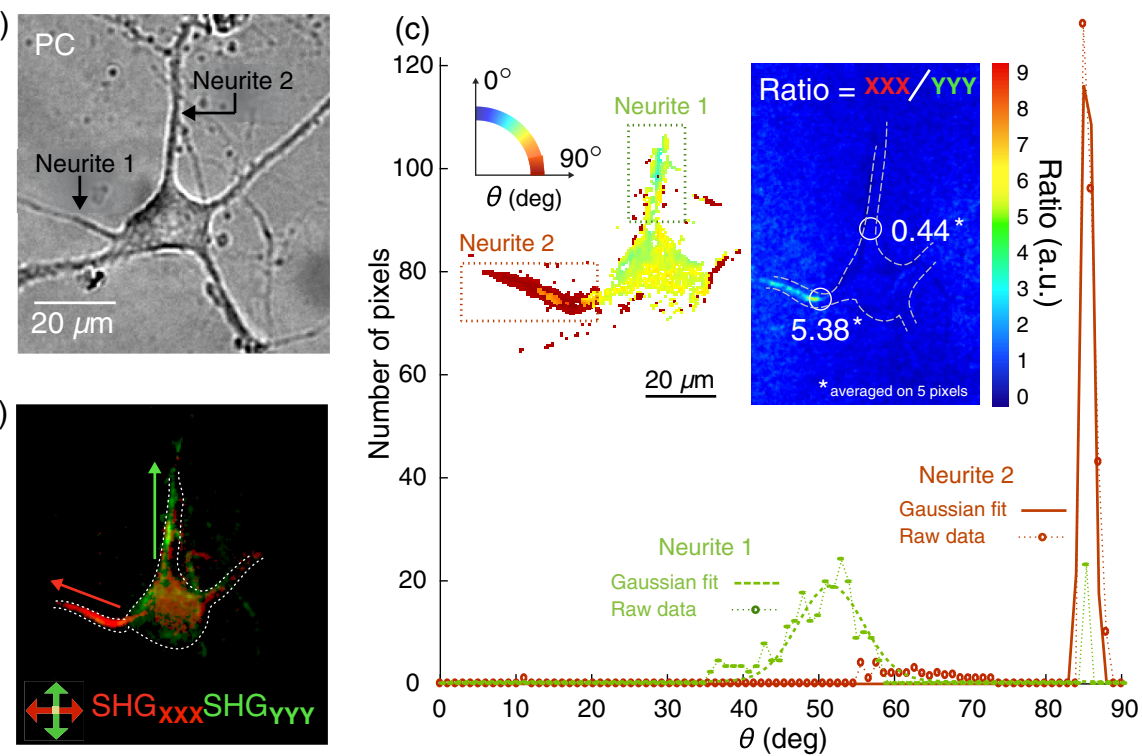

Fig. 2 The orientational distribution of microtubules from SH polarimetry. (a) PC image of a neuron DIV13. The neuron consists of a cell body and two neurites. (b) SH images recorded in XXX (red) and YYY (green) polarization combinations. The purely imaging acquisition time of this image was $18 \mathrm{~s}$ for each polarization without considering the total time, including the manual manipulation of the waveplate. (c) The orientational distribution of the neurites is plotted relative to the main axis of the neurite with their respective Gaussian fits. The inset shows a color plot indicating the pixel-wise relative tilt angle in degrees obtained from applying Eq. (3) to the data in (b).

Gaussians [Fig. 2(c)] that have standard deviations of $0.76 \mathrm{deg}$ (neurite 1) and $4.3 \mathrm{deg}$ (neurite 2), respectively. The inset of Fig. 2(c) shows a color map with the computed orientational angles per pixel, with the $Y$ direction set to $0 \mathrm{deg}$ and the $X$ direction set to $90 \mathrm{deg}$.

Of all the microtubule-containing structures within the cell, the axon is the only one that presents a unidirectional organized array of microtubules, oriented along the growth direction and arranged in parallel ordered bundles. ${ }^{37,44-46}$ Based on the above analysis, we can thus conclude that the structure that has a high $\mathrm{SH}$ intensity in combination with a narrow orientational distribution that is directed parallel to the growth direction is the axon (neurite 1), while the other one is a dendrite (neurite 2).

\subsection{Second-Harmonic Imaging of Microtubule Morphology in Single Neurons}

Next, we measure the influence of nocodazole on the SH intensity. Figure 3(a) shows a composite image obtained with PC and SH modalities showing two morphologically polarized neurons at DIV7. At this stage of maturity, neurons have one long and thin process, the axon, and several shorter tapered processes. ${ }^{47,48}$ This neuronal morphology is observed in the PC image in Fig. 3(a). The SH signal, in red in Fig. 3(a), was obtained with the two incoming beams polarized along the vertical direction (YYY, indicated by the red double-headed arrow). It can be seen that one of the structures in Fig. 3(a) generates a brighter SH signal (indicated by the white arrow), while other structures, such as the cell bodies (indicated by the white arrowheads) emit a much fainter SH intensity. This difference in intensity already indicates that the brighter structure is likely the axon. For the axon, an excitation parallel to the microtubule molecular dipole axis leads to a strong $\mathrm{SH}$ emission, while excitation perpendicular to this axial direction should result in no $\mathrm{SH}$ contrast. Changing the polarization combination of the incoming and outgoing beams, we only observe an intense $\mathrm{SH}$ emission from the structure highlighted by the white arrow. Thus, the elongated structure in Fig. 3(a) most likely corresponds to the axon.

Nocodazole depolymerizes microtubules and should therefore affect the SH intensity, in accordance with previous studies $^{26}$ that showed that $\mathrm{SH}$ depletion and recovery follows the application of the depolymerizing drug nocodazole. To investigate the effects of nocodazole on the SH intensity, we applied a $10-\mu \mathrm{M}$ nocodazole solution in the chamber for $9 \mathrm{~min}$ and subsequently washed it out. SH images were recorded continuously with $0.25 \mathrm{~s}$ per frame. The histograms in Fig. 3(b) show the 2.5 -s-averaged SH intensity in counts coming from the neurons marked by the dashed masks in Fig. 3(a) before and after the application of nocodazole and at the end of the recovery time. It can be seen that after 9 min of drug exposure, the $\mathrm{SH}$ intensity arising from the two cells decreases to $\sim 60 \%$ of its original value. After $20 \mathrm{~min}$ following the washout, the $\mathrm{SH}$ intensity has recovered back to $\sim 80 \%$ of its original value, in agreement with previous studies. ${ }^{26,37}$ As the wide-field $\mathrm{SH}$ imaging system can be employed to record 0.25-s integration time images, without damaging the cells, ${ }^{43}$ in what follows we demonstrate two possible applications of $\mathrm{SH}$ imaging: to probe morphological drug-induced changes in neurons of different age and to dynamically image spatiotemporal changes in real time.

\subsection{Mapping Changes in the Microtubule Morphology as a Function of Maturity}

Figure 4(a) shows PC and SH images of neurons at three different stages of maturity: DIV7, DIV11, and DIV23. For each neuron, the displayed SH intensity is averaged over $10 \mathrm{~s}$. Figure 4(b) 
(a)

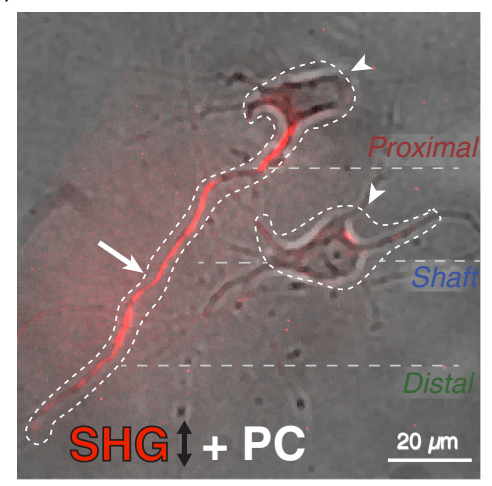

(b)

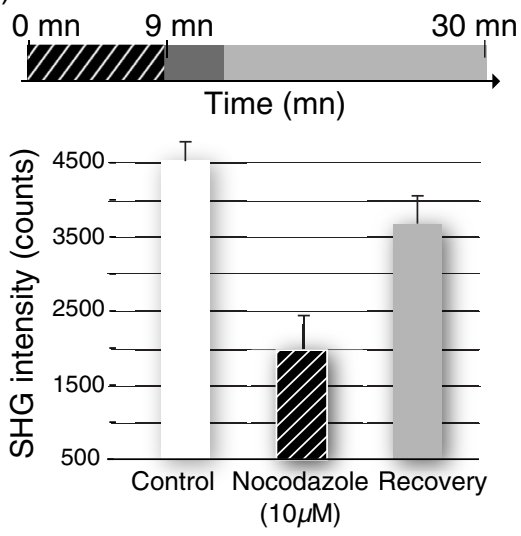

Fig. 3 Effect of nocodazole on the SH response from microtubules, during application and after washout. (a) Composite of the nonpolarized white-light PC and SH (red color) image of two neurons at stage 3 of their development. The polarization combination used for the $\mathrm{SH}$ image is indicated by the red doubleheaded arrow. Arrowheads: cell bodies, arrow: axon. The axon is divided into three parts, proximal, shaft, and distal, which will be used for the spatiotemporal SH imaging using the same neuron in Fig. 5. (b) Top panel: time lapse protocol of the nocodazole application in the washout and recovery experiment. Nocodazole $(10 \mu \mathrm{M})$ was added to the open bath chamber containing the plated neurons (striped shading); 9 min later the drug-containing solute was removed and the culture was washed three times with drug-free culture medium (dark gray shading). Recovery lasts $20 \mathrm{~min}$ after the washout (light gray shading). Bottom panel: histograms of the overall SH intensity from the dashed lines in (a) averaged for $3 \mathrm{~s}$ during the control (no drug added), directly after injection of nocodazole and after a recovery period of $20 \mathrm{~min}$.

(a)

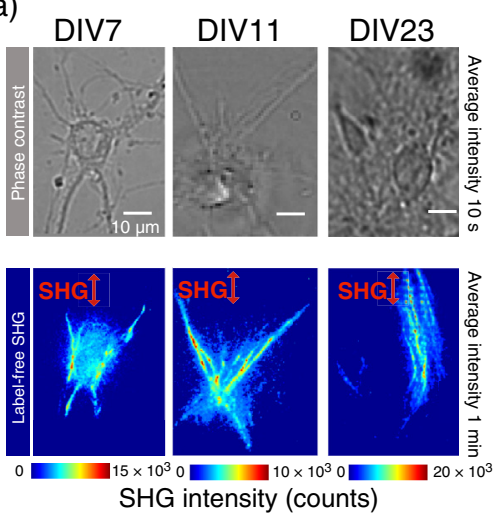

(b) $\quad$ Time (mn)

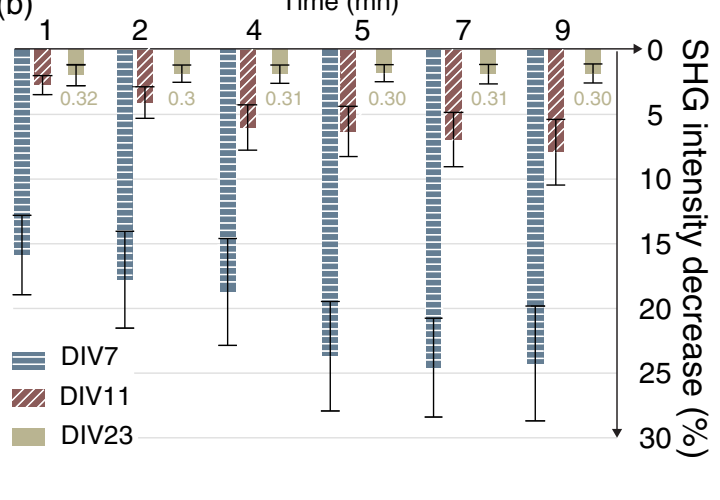

Fig. 4 Nocodazole-induced SH intensity depletion from the cytoskeleton of neurons at different stages of maturity. (a) Top panels: PC images of cultured neurons at different stage of maturity. Bottom panels: respective label-free SH images averaged over $10 \mathrm{~s}$ during the control conditions. (b) Histograms of $\mathrm{SH}$ intensity decreases (in percentage) as a function of the time of exposure to a $10-\mu \mathrm{g} / \mathrm{ml}$ solution of nocodazole.

shows the decrease in signal after application of nocodazole where the first $10 \mathrm{~s}$ were taken as a reference (before nocodazole application). Figure 4(b) shows that newly polarized neurons (DIV7) show a strong response to nocodazole, with an $\mathrm{SH}$ depletion of $15 \%$ after $1 \mathrm{~min}$ and a decrease down to $25 \%$ of the original value after $9 \mathrm{~min}$. Mature polarized neurons (DIV11) display a slow decrease over a long time, starting from $2.7 \%$ after 1 min with an SH intensity depletion of $8 \%$ of its original value after $9 \mathrm{~min}$ of exposure. For advanced mature neurons (DIV23), the SH signal is not changing detectably. This difference in nocodazole sensitivity with age is consistent with a dynamical microtubule cytoskeleton, where at the early stages more and shorter microtubules are present that have many sites to initiate depolymerization. As neurons mature, microtubules are more inclined to collect post-translational modifications, resulting in an increase of stability. ${ }^{49,50}$ Thus, the microtubules of the cytoskeleton of older neurons have a better resistance to nocodazole. Since wide-field SH imaging allows for time lapse imaging over long time intervals, one could potentially use this method to follow the same neuron over extended periods of time in future experiments.

\subsection{In Vitro Second-Harmonic Imaging of Multisite Time-Resolved Microtubule Morphology Changes}

In another application, we demonstrate the possibility of dynamic SH imaging to obtain spatiotemporal stability information about the axonal microtubule network. We image the microtubule network of a single axon from a newly polarized neuron 

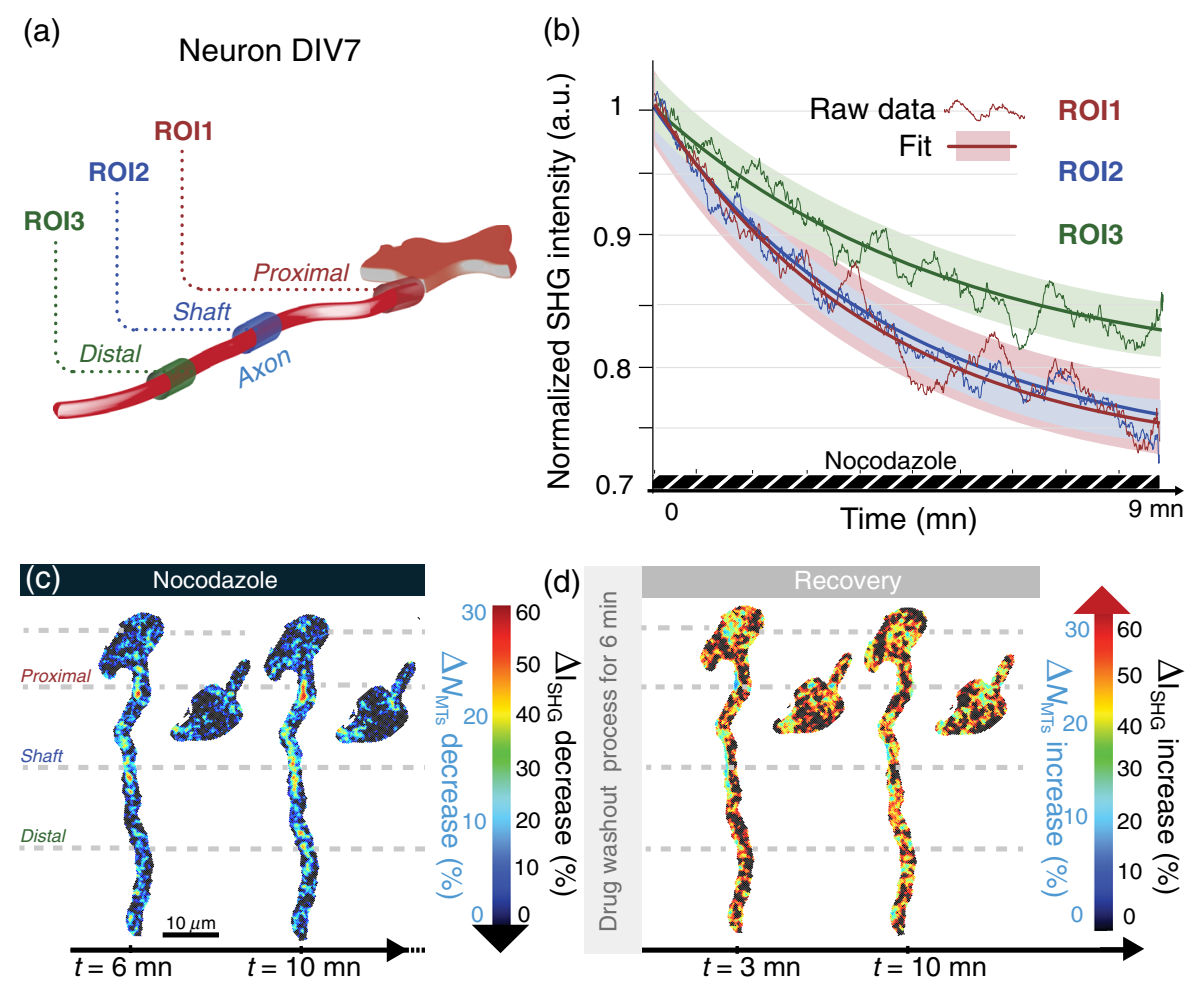

Fig. 5 Spatiotemporal SH imaging of microtubule stability. (a) Schematic view of an axon with three different ROIs. (b) Normalized nocodazole-induced SH intensity depletion for the three different ROIs indicated in (a). The colored intensity traces represent the filtered raw data, obtained with a seventhorder median filter, the smoothed darker color traces represent intensity traces that were filtered with a 100th-order median filter and a low pass moving-average filter with a span of 5. (c) Spatiotemporal maps of percentage of decrease in $\mathrm{SH}\left(\Delta /_{\mathrm{SHG}}\right)$ and microtubule density $\left(\Delta N_{\mathrm{MTS}}\right)$. The control signal is taken at $t=0$ min when the drug is injected into the bath (average of 10 frames with $0.25 \mathrm{~s} /$ frame). The decrease in the $\mathrm{SH}$ intensity and microtubules density is mapped as a function of time during the $10 \mathrm{~min}$ of nocodazole treatment $(10 \mu \mathrm{g} / \mathrm{mL})$ and after $20 \mathrm{~min}$ of recovery following the washout. The gray dashed lines correspond to different morphological areas along the axon: proximal, shaft (middle shaft of the axon), and distal (distal axonal shaft). In the proximal region of the axon, we observed a gradual loss of $\mathrm{SH}$ intensity spreading toward the distal part of the axon. During the recovery, the middle axonal shaft shows a spread damaged area of $\mathrm{SH}$ loss recovering toward the proximal part in discrete local points.

[DIV7, the cell depicted in Figure 3(a)] treated with nocodazole and after washout. We examine the SH intensity variations in the 0.25 -s acquisition time $\mathrm{SH}$ images and process the intensity variations that occur along three different regions of the neuron in Fig. 3(a) and represented in the schematic of Fig. 5(a): the proximal region closest to the cell body [region of interest (ROI) 1], a middle shaft part (ROI 2, >50 $\mu \mathrm{m}$ from the cell body), and a distal shaft part (ROI 3, >100 $\mu \mathrm{m}$ from the cell body). Figure 5(b) shows the decay of the averaged SH intensity as a function of time during nocodazole application for the three ROIs. Single exponential decays are used to fit the data. These fits show that during nocodazole exposure, an exponential depletion in the SH intensity is observed as a function of time, with different decay times for three ROIs along the axon: the averaged $\mathrm{SH}$ intensity from the proximal region decreases with a half-time of $2 \min 30 \mathrm{~s} \pm 35 \mathrm{~s}\left(R^{2}=0.93\right)$, whereas the halftimes of the middle and distal axonal shafts are slower, $2 \mathrm{~min}$ $48 \mathrm{~s} \pm 25 \mathrm{~s}\left(R^{2}=0.98\right)$ and $3 \min 47 \mathrm{~s} \pm 76 \mathrm{~s}\left(R^{2}=0.95\right)$, respectively. We observe that the half-time values of the $\mathrm{SH}$ intensity depletion during nocodazole treatment increase with respect to the distance from the cell body. These observations are in agreement with literature ${ }^{51}$ although the optical contrast mechanism is dissimilar; the similarity in decay times during nocodazole exposure suggests that both methods report on the decrease in the density of axonal microtubules.

In addition to analyzing the spatially averaged decay of the SH intensity in three distinct ROIs, we make a pixel-wise comparison and map the reduction and the recovery of SH intensity per pixel as a function of time for the whole neurons [Figs. 5(c)5(d)] and look at the SH intensity variations in the axon. The directional polarity of microtubules in the axon is uniform, ${ }^{7}$ $\left(P_{\mathrm{MT}}=1\right)$, as is confirmed in Fig. 2(c) that showed the very narrow orientational distribution in neurite 1 . This specific uniform polarity allows us to relate the $\mathrm{SH}$ intensity to the number of uniformly oriented microtubules ${ }^{35}$ with $I_{\mathrm{SHG}} \propto$ $\left(N_{\mathrm{MT}} \times P_{\mathrm{MT}}\right)^{2}$. Applying Eqs. (1)-(3) with the assumption that nocodazole does not alter the orientational distribution of the microtubules, we can relate the relative $\mathrm{SH}$ intensity to the relative change in the number of microtubules during and after nocodazole application:

$$
\frac{N_{\mathrm{MTs}}(t)}{N_{\mathrm{MTs}, 0}}=\sqrt{\frac{I_{\mathrm{SH}}(t)}{I_{\mathrm{SH}, 0}}},
$$


where $I_{\mathrm{SH}, 0}$ denotes the initially measured $\mathrm{SH}$ intensity of the axon and $N_{\mathrm{MT}, 0}$ denotes the corresponding initial number of microtubules at $t=0$ before adding nocodazole. Since Baas has shown in Ref. 52 that after nocodazole treatment the microtubule polarity is for $96 \%$ identical to the pretreated neuron, we think the above assumption is reasonable. With this approach, it is possible to map on a pixel-size scale the spatiotemporal fluctuations of $\mathrm{SH}$ intensity and the corresponding variation in the number of microtubules. Figure 5(c) shows a map of the percentage of decrease in SH intensity (black scale, $\left.\Delta I(t)=\left[I_{\mathrm{SH}, 0}-I_{\mathrm{SH}}(t)\right] \times 100 / I_{\mathrm{SH}, 0}\right)$ and corresponding relative change in the number of microtubules (blue scale, $\left.\Delta N_{\mathrm{MTs}}(t)=\left[\sqrt{I_{\mathrm{SH}, 0}}-\sqrt{I_{\mathrm{SH}}(t)}\right] \times 100 / \sqrt{I_{\mathrm{SH}, 0}}\right)$ during nocodazole exposure. Figure 5(d) shows the relative $\mathrm{SH}$ intensity increase and converted increase in the relative number of microtubules during recovery. It can be seen that $6 \mathrm{~min}$ after the nocodazole application, there are isolated sites $\sim 1 \mu \mathrm{m}$ in size that have a $30 \%$ reduction in the number of axonal microtubules. These sites occur more frequently closer to the cell body. In the proximal part, there is also a $3-\mu \mathrm{m}$-size core with a similar microtubule density decrease. After $9 \mathrm{~min}$, these discrete sites of damage connect and expand toward the distal part of the axonal shaft. During the recovery, the damaged cores are restored, whereby the distal part appears to heal the quickest.

Thus, the above data demonstrate the possibility of imaging spatiotemporal changes in vitro during and after the application of the microtubule-destabilizing drug nocodazole. Since the presented data set is very limited $(N=7)$, it is impossible to draw conclusions about the mechanistic effect of nocodazole. Future more extensive studies could map the degradation more clearly as well as conduct intermediate real-time polarimetric measurements to determine how and where the microtubule network undergoes structural changes. A combination with $\mathrm{SH}$ interferometry ${ }^{36}$ could add information on microtubule directionality. Combined SH and fluorescence or electron microscopy studies are also potentially powerful tools to increase our knowledge of the complex microtubule network within neurons and other cells.

\section{Conclusions}

Wide-field, high-throughput SH microscopy was applied to study living mammalian neurons with the aim of demonstrating the possibility to extract the orientational distribution of microtubules as well as obtaining dynamic label-free spatiotemporal stability (referred as resistance to the drug nocodazole) information in vitro. We used nocodazole, a microtubule-destabilizing drug, as it is a well-known reversible microtubule-specific depolymerizing drug. We first introduced the principal physical relations needed to understand $\mathrm{SH}$ imaging. We performed $\mathrm{SH}$ polarimetry measurements to demonstrate how the pixel-wise orientational directionality of microtubules in neurites and cell bodies can be determined. When the microtubules are oriented along the principle axis of the neurite with a narrow orientational distribution (rather than having a broad orientational distribution that is not aligned with the principle axis of the neurite) and when the locally emitted SH intensity is significantly higher than in other parts, we can assign a structure as the axon. We also showed the possibility of extracting information about the stability of the cytoskeleton against nocodazole-induced depolymerization as a function of the neurons age in vitro with $\mathrm{SH}$ imaging. We then performed spatiotemporal SH imaging during and after the application of nocodazole and focused our observations on a single axon of a morphologically polarized cultured neuron. Assuming that nocodazole does not affect the orientational distribution of microtubules, direct statements can be made to relate the variations in the $\mathrm{SH}$ intensity with the changes in microtubules density.

These measurements show the possibility of real-time in vitro imaging of changes in the density of the axonal microtubules. In combination with other techniques, our quantitative understanding of microtubule cytoskeletal dynamics could be enhanced considerably, especially since the presented approach is noninvasive and can be performed in real-time and label-free. This work constitutes a state-of-the-art example of the imaging and quantitative possibilities our wide-field, label-free, SH technique offers. Future investigations could lead to further applications such as the investigation of neurodegenerative diseases or spatiotemporal dynamics studies of mitosis.

\section{Disclosures}

No conflicts of interest, financial or otherwise, are declared by the authors.

\section{Acknowledgments}

The authors would like to thank E. Ruchti, E. Gasparetto, and M. Wirth for providing the neuronal cultures, and I. Allaman and L. Kapitein for discussions. This work was supported by the Julia Jacobi Foundation, the Swiss National Foundation (Grant No. 200021_146884), and the European Commission Research Executive Agency Marie Curie Actions "FINON" (Grant No. ITN-2013-607842).

\section{References}

1. T. Mitchison and M. Kirschner, "Dynamic instability of microtubule growth," Nature 312, 237-242 (1984).

2. R. L. Margolis and L. Wilson, "Opposite end assembly and disassembly of microtubules at steady state in vitro," Cell 13, 1-8 (1978).

3. E. D. Salmon, M. McKeel, and T. Hays, "Rapid rate of tubulin dissociation from microtubules in the mitotic spindle in vivo measured by blocking polymerization with colchicine," J. Cell Biol. 99, 1066-1075 (1984).

4. P. W. Baas, "Microtubules and neuronal polarity: lessons from mitosis," Neuron 22, 23-31 (1999).

5. J. M. Gibbs-Davis et al., "Jammed acid-base reactions at interfaces," J. Am. Chem. Soc. 130, 15444-15447 (2008).

6. P. Janmey, Handbook of Biological Physics, R. Lipowsky and E. Sackmann eds., Vol. 1, pp. 805-849, Elsevier, North Holland (1995).

7. J. W. Mandell and G. A. Banker, "The microtubule cytoskeleton and the development of neuronal polarity," Neurobiol. Aging 16, 229-237; discussion 238 (1995)

8. T. Yamaguchi, K. Nishizaki, and S. Itai, "Molecular interactions between phospholipids and electrolytes in a monolayer of parenteral lipid emulsion," Colloids Surf. B Biointerfaces 9, 275-282 (1997).

9. K. W. Yau et al., "Dendrites in vitro and in vivo contain microtubules of opposite polarity and axon formation correlates with uniform plusend-out microtubule orientation," J. Neurosci. 36, 1071-1085 (2016).

10. J. D. Wilson et al., "Light scattering from intact cells reports oxidativestress-induced mitochondrial swelling," Biophys. J. 88, 2929-2938 (2005).

11. S. H. Brawley and R. S. Quatrano, "Effects of microtubule inhibitors on pronuclear migration and embryogenesis in Fucus distichus (phaeophyta)," J. Phycol. 15, 266-272 (1979).

12. J. Hoebeke, G. Van Nijen, and M. De Brabander, "Interaction of oncodazole (R 17934), a new anti-tumoral drug, with rat brain tubulin," Biochem. Biophys. Res. Commun. 69, 319-324 (1976).

13. H. Witte, D. Neukirchen, and F. Bradke, "Microtubule stabilization specifies initial neuronal polarization," J. Cell Biol. 180, 619-632 (2008). 
14. L. Wilson and M. A. Jordan, "Microtubule dynamics: taking aim at a moving target," Chem. Biol. 2, 569-573 (1995).

15. J. C. Lee, D. J. Field, and L. L. Lee, "Effects of nocodazole on structures of calf brain tubulin," Biochemistry 19, 6209-6215 (1980).

16. T. Horio and H. Hirokazu, "Visualization of the dynamic instability of individual microtubules by dark-field microscopy," Nature 319, 402-405 (1986).

17. T. Haraguchi, T. Kaneda, and Y. Hiraoka, "Dynamics of chromosomes and microtubules visualized by multiple-wavelength fluorescence imaging in living mammalian cells: effects of mitotic inhibitors on cell cycle progression," Genes Cells 2, 369-380 (1997).

18. P. M. Bayley, M. J. Schilstra, and S. R. Martin, "Microtubule dynamic instability: numerical simulation of microtubule transition properties using a lateral cap model," J. Cell Sci. 95, 33-48 (1990).

19. P. J. Sammak and G. G. Borisy, "Direct observation of microtubule dynamics in living cells," Nature 332, 724-726 (1988).

20. T. Stepanova et al., "History-dependent catastrophes regulate axonal microtubule behavior," Curr. Biol. 20, 1023-1028 (2010).

21. D. R. Webster et al., "Differential turnover of tyrosinated and detyrosinated microtubules," Proc. Natl. Acad. Sci. U. S. A. 84, 9040-9044 (1987).

22. N. M. Rusan et al., "Cell cycle-dependent changes in microtubule dynamics in living cells expressing green fluorescent protein-alpha tubulin," Mol. Biol. Cell 12, 971-980 (2001).

23. P. Bon et al., "Fast label-free cytoskeletal network imaging in living mammalian cells," Biophys. J. 106, 1588-1595 (2014).

24. U. Marklund et al., "Oncoprotein 18 is a phosphorylation-responsive regulator of microtubule dynamics," EMBO J. 15, 5290-5298 (1996).

25. C. Macias-Romero and P. Török, "Eigenvalue calibration methods for polarimetry," J. Eur. Opt. Soc. 7, 1990-2573 (2012).

26. D. A. Dombeck et al., "Uniform polarity microtubule assemblies imaged in native brain tissue by second-harmonic generation microscopy," Proc. Natl. Acad. Sci. U. S. A. 100, 7081-7086 (2003).

27. B. F. Levine and C. G. Bethea, "Second and third order hyperpolarizabilities of organic molecules," J. Chem. Phys. 63, 2666-2682 (1975).

28. W. H. Stoothoff, B. J. Bacskai, and B. T. Hyman, "Monitoring tautubulin interactions utilizing second harmonic generation in living neurons," J. Biomed. Opt. 13, 064039 (2008).

29. W. R. Boyd, Nonlinear Optics, Academic Press (1992).

30. H. Goldstein, C. P. Poole, and J. L. Safko, Classical Mechanics, 3rd ed., Pearson, Harlow (2001).

31. D. Sharoukhov and H. Lim, "On probing conformation of microtubules by second-harmonic generation," J. Mod. Opt. 63, 71-75 (2016).

32. A. E. Tuer et al., "Hierarchical model of fibrillar collagen organization for interpreting the second-order susceptibility tensors in biological tissue," Biophys. J. 103, 2093-2105 (2012).

33. S. Psilodimitrakopoulos et al., "Estimation of the effective orientation of the SHG source in primary cortical neurons," Opt. Express 17, 14418-14425 (2009).

34. C. Odin et al., "Second harmonic microscopy of axonemes," Opt. Express 17, 9235-9240 (2009).

35. C.-H. Yu et al., "Measuring microtubule polarity in spindles with second-harmonic generation," Biophys. J. 106, 1578-1587 (2014).

36. S. Bancelin et al., "Probing microtubules polarity in mitotic spindles in situ using interferometric second harmonic generation microscopy," Sci. Rep. 7, 1-8 (2017).

37. A. C. Kwan, D. A. Dombeck, and W. W. Webb, "Polarized microtubule arrays in apical dendrites and axons," Proc. Natl. Acad. Sci. U. S. A. 105, 11370-11375 (2008).
38. W. Denk, J. H. Strickler, and W. W. Webb, "Two-photon laser scanning fluorescence microscopy," Science 248, 73-76 (1990).

39. P. J. Campagnola et al., "High-resolution nonlinear optical imaging of live cells by second harmonic generation," Biophys. J. 77, 3341-3349 (1999).

40. X. Chen et al., "Second harmonic generation microscopy for quantitative analysis of collagen fibrillar structure," Nat. Protoc. 7, 654-669 (2012).

41. C. Macias-Romero et al., "Optical imaging of surface chemistry and dynamics in confinement," Science 357, 784-788 (2017).

42. C. Macias-Romero et al., "High throughput second harmonic imaging for label-free biological applications," Opt. Express 22, 31102-31112 (2014).

43. C. Macias-Romero et al., "Wide-field medium-repetition-rate multiphoton microscopy reduces photodamage of living cells," Biomed. Opt. Express 7, 1458-1467 (2016).

44. K. Kollins et al., "Dendrites differ from axons in patterns of microtubule stability and polymerization during development," Neural Dev. 4, 26 (2009).

45. C. Conde and A. Cáceres, "Microtubule assembly, organization and dynamics in axons and dendrites," Nat. Rev. Neurosci. 10, 319-332 (2009).

46. P. W. Baas and S. Lin, "Hooks and comets: the story of microtubule polarity orientation in the neuron," Dev. Neurobiol. 71, 403-418 (2011).

47. E. Kandel, J. Schwartz, and T. Jessell, Principles of Neural Science, McGraw-Hill Medical, New York (2013).

48. A. M. Craig and G. Branker, "Neuronal polarity," Cold Spring Harb. Perspect. Biol. 1, a001644 (1994).

49. J. Bulinski and G. Gundersen, "Stabilization of post-translational modification of microtubules during cellular morphogenesis," BioEssays News Rev. Mol. 13, 285-293 (1991).

50. G. Piperno, M. LeDizet, and X. J. Chang, "Microtubules containing acetylated alpha-tubulin in mammalian cells in culture," J. Cell Biol. 104, 289-302 (1987).

51. M. Kempf et al., "Tau binds to the distal axon early in development of polarity in a microtubule- and microfilament-dependent manner," J. Neurosci. 16, 5583-5592 (1996).

52. P. W. Baas, M. M. Black, and G. A. Banker, "Changes in microtubule polarity orientation during the development of hippocampal neurons in culture," J. Cell Biol. 109, 3085-3094 (1989).

Marie E. P. Didier obtained her two MSc degrees in conservation science from the University of Bordeaux and in physics from Paris 6. She obtained her PhD in nonlinear optics applied to neurophotonics from École Polytechnique Fédérale de Lausanne in 2018. She has a pluridisciplinary background with proven ability to develop innovative nondestructive imaging technologies to characterize fragile samples either in biology or in art (varnish, paintings). She is an excellent communicator and naturally thrives in an interdisciplinary environment.

Sylvie Roke obtained her MSc degrees with highest honors in chemistry and physics from Utrecht University in 2000 and her $\mathrm{PhD}$ in natural sciences from Leiden University in 2004, highest honors. In 2005 she was awarded an independent research group leader (W2) position by the Max Planck Society. In 2011 she became director of the Laboratory for fundamental BioPhotonics at EPFL, and holder of the Julie Jacobi Chair in photomedicine.

Biographies of the other authors are not available. 